\title{
La atención sostenida como una desigualdad en el desempeño académico
}

\section{Sustained attention as inequality in academic performance}

1 Verónica Andreina Caizaguano Azogue

Universidad Técnica de Ambato, Facultad de Ciencias de la Educación, Carrera de psicopedagogía. Ambato, Ecuador vcaizaguano2647@uta.edu.ec

2 Roberto Enrique Alvarado Quinto Universidad Técnica de Ambato, Facultad de Ciencias de la Educación, Carrera de psicopedagogía. Ambato, Ecuador re.alvarado@uta.edu.ec

3 Fredy Aguilar Rodríguez Universidad Técnica de Ambato, Facultad de Ciencias sociales, Carrera de comunicación. Ambato, Ecuador.

wf.aguilar@uta.edu.ec

4 Rodrigo Andrade Alban https://orcid.org/0000-0002-8743-9

Universidad Técnica de Ambato, Facultad de Ciencias de la Educación, Carrera de Psicopedagogía. Ambato, Ecuador jr.andrade@uta.edu.ec

Artículo de Investigación Científica y Tecnológica Enviado: 24/12/2021

Revisado: $29 / 12 / 2021$

Aceptado: 12/01/2022

Publicado:08/03/2023

DOI: https://doi.org/10.33262/concienciadigital.v6i1.4.2055

Cítese: Caizaguano Azogue, V. A., Alvarado Quinto, R. E., Aguilar Rodríguez, F., \& Andrade Alban, R. (2023). La atención sostenida como una desigualdad en el desempeño ConcienciaDigital, 1098-1112. https://doi.org/10.33262/concienciadigital.v6i1.4.2055

CONCIENCIA DIGITAL, es una Revista Multidisciplinar, Trimestral, que se publicará en soporte electrónico tiene como misión contribuir a la formación de profesionales competentes con visión humanística y crítica que sean capaces de exponer sus resultados investigativos y científicos en la misma medida que se promueva mediante su intervención cambios positivos en la sociedad. https://concienciadigital.org

La revista es editada por la Editorial Ciencia Digital (Editorial de prestigio registrada en la Cámara Ecuatoriana de Libro con No de Afiliación 663) www.celibro.org.ec 


\section{Palabras} claves:

atención

sostenida, desempeño académico, test de atención d2
Keywords:

sustained

attention,

academic

performance, attention test $\mathrm{d} 2$
Resumen

En la presente investigación con el tema la atención sostenida como una desigualdad en el desempeño académico se ha planteado como objetivo el análisis del rendimiento que presentan los estudiantes universitarios en las dimensiones de la prueba de atención d2 teniendo en cuenta los diferentes estímulos distractores que intervienen en esta nueva modalidad de educación online. La metodología que se utilizó en esta investigación es de tipo cuantitativa y cualitativa, ya que permitió obtener datos estadísticos de los instrumentos aplicados y de la misma manera su interpretación. Los instrumentos aplicados son la prueba de atención d2 por Rolf Brinckenkamp y un cuestionario para el desempeño académico validado por un profesional experto. Se evaluó a 110 estudiantes universitarios los cuales 28 son hombres y 82 son mujeres entre 18- 24 años. Los resultados obtenidos en el primer instrumento fue una baja precisión en la identificación de estímulos relevantes, poca atención y omisión de respuestas correctas, un nivel bajo en la discriminación de estímulos relevantes, fatiga atencional, mal rendimiento en la atención sostenida e ineficiencia en la realización de una tarea. En el segundo instrumento se pudo evidenciar dificultades en el mantenimiento de la atención por largos periodos de tiempo debido a los diferentes estímulos distractores que se presentan durante la jornada de clases y las estrategias pedagógicas que usan los docentes para impartir los contenidos de las materias los mismos que traen consecuencias en su desempeño académico.

\section{Abstract}

In the present investigation with the subject of sustained attention as inequality in academic performance, the objective of analyzing the performance presented by university students in the dimensions of the Attention Test $\mathrm{d} 2$ has been established, considering the different distracting stimuli that intervene in this new form of online education. The methodology used in this research is quantitative and qualitative, since it allowed obtaining statistical data from the applied instruments and, in the same way, their interpretation. The instruments applied are the Rolf Brinckenkamp Attention Test d2 and an academic performance questionnaire validated by an expert professional. 110 university students were evaluated, 28 men and 82 women between 18-24 years old. The results obtained in the first 
instrument were a low precision in the identification of relevant stimuli, little attention and omission of correct responses, a low level in the discrimination of relevant stimuli, attentional fatigue, poor performance in sustained attention and inefficiency in performance. of a task. The second instrument showed difficulties in maintaining attention for long periods of time due to the different distracting stimuli that are presented during the class day and the pedagogical strategies used by teachers to teach the contents of the subjects themselves that have consequences about their academic performance.

\section{Introducción}

\section{La atención}

Flores (2016) define a la atención como la capacidad de mantener la vigilancia de forma voluntaria por largos periodos de tiempo en un fenómeno de la realidad. El control de la atención se evidencia en el manejo de sus cualidades, basándose en la necesidad de cada individuo cuando se encuentran ante el proceso de aprendizaje permitiendo direccionarlos hacia sus intereses.

La atención está relacionada con términos como concentración, estado de alerta, interés, percepción, memoria y reacción que son las respuestas que se obtienen de esta acción. La atención inicia con el acto de percibir mediante los órganos sensoriales (visión, audición, olfato, tacto y gusto), sin embargo, en su mayoría la atención de un individuo se da mediante la visión ya que la presencia de estímulos iluminados, grandes, colores llamativos o incluso objetos y situaciones atraerá la atención de cualquiera al momento de percibirlo. Se puede notar la eficiencia de la atención cuando un estímulo es bien percibido y analizado mientras que el resto se ubica en un papel secundario. La atención se entrena y estimula a nivel de funciones cognitivas intelectuales debido a que están encargadas de dar prioridad y orden de importancia a los procedimientos intelectuales para dar respuestas claras (Boujon \& Quaireau, 2004).

Mediante una revisión en los aportes teóricos de Posner y Petersen (1990) sobre la atención se encontró tres diferentes modelos de redes atencionales; la red de alerta, la red ejecutiva y finalmente la red de orientación.

\section{Modelo de los componentes atencionales}

El modelo denominado red de alerta se relaciona con mantener el estado de guardia o atención ante un estímulo y generar una respuesta ante el mismo. Existen algunos 
términos que se pueden utilizar para referirse al mantenimiento de la atención, por ejemplo; vigilancia, y atención sostenida. Su definición corresponde a la capacidad y eficacia cognitiva para mantener un nivel alto en los sistemas atencionales los mismos que pueden incrementarse con la ayuda del control ejecutivo. Para llevar a cabo esta función requiere la participación e integración de la formación reticular y la red corticosubcotical lateral que está encargada de mantener el nivel de arousal correcto; además de producir proyecciones noradrenérgicas partiendo del locus coeruleus hacia el tálamo y prosencéfalo basal; asimismo el córtex cingulado anterior está encargado del mantenimiento de la atención voluntaria (Raz \& Buhle, 2006).

La red de orientación es aquella que hace la selección específica de un estímulo entre otros; por lo mismo hace énfasis a la atención selectiva que trata de explicar el proceso de seleccionar la información relevante entre los estímulos irrelevantes los cuales serán inhibidos (Posner \& Petersen, 1990).

En el modelo de control atencional Corbetta y Shulman (2002) mencionan los mecanismos de atención endógena o también llamada activación de mecanismos de arriba hacia abajo los cuales se dirigen por la representación mental, los objetivos y conocimientos, los mismos que tienen referencia con la red de orientación. Así mismo la atención exógena que esta direccionada a la estimulación sensorial percibida del ambiente externo.

Existe una interacción entre la atención endógena y un estímulo externo inesperado o novedoso que esta intervenido por factores internos como las instrucciones dadas con anterioridad, el conocimiento o experiencia antes almacenada (Posner \& Petersen, 1990).

Este modelo no se refiere solo a la orientación sino además del control ya que mantienen una constante interacción para determinar a lo que ponemos atención, como lo hacemos y donde lo hacemos. Estas formas de control atencional se localizan en las redes neuronales independientes que mediante estudios en lesiones y de comportamiento se ha demostrado que pueden desligarse, por el contrario, se ha encontrado que esto no sucede en los estudios de neuroimagen (Nobre, 2001).

Finalmente, la red ejecutiva está encargada de la planificación, detección, toma de decisiones, preparación de respuestas, distribución de recursos atencionales, supervisión de conductas y regulación de pensamientos. La activación de la red ejecutiva se da en momentos en que se requieren el procesamiento de información o el control eficaz del comportamiento. En estas funciones se encuentran involucradas diferentes áreas cerebrales en gran parte de los lóbulos prefrontales juntamente con estructuras subcorticales. Las regiones del córtex cingulado anterior se encargan del manejo de conflictos, detección de errores, ejecución de la tarea y la atención dividida. Así mismo el córtex prefrontal lateral es el responsable de crear y mantener los componentes 
atencionales como la memoria operativa. Por último, el córtex frontal inferior que se activan con la presencia de estímulos de baja frecuencia, además de estar conectados con las regiones temporoparietales permiten la atención exógena denominada a la capacidad de percibir un estímulo ambiental (Campabadal et al., 2008).

\section{Cualidades de la atención}

La atención está conformada por las siguientes cualidades según Luria (1979):

- Distribución de la atención: se denomina a la capacidad de mantener el foco atencional en distintos estímulos por un periodo de tiempo considerable.

- Oscilación de la atención: se denomina a la capacidad de cambiar la focalización de la atención de un objetivo a otro de manera involuntaria.

- Estabilidad de la atención: se refiere principalmente a la consistencia atencional en una tarea por un periodo de tiempo largo que permite profundizar la información.

- Volumen de la atención: se denomina a la capacidad de procesar con claridad las tareas mostradas basándose en la experiencia.

- $\quad$ Intensidad de la atención: se refiere a la cantidad de atención proporcionada al estímulo para mantener una extremada concentración.

\section{Tipología de la atención}

Según los mecanismos implicados por Esparza y Casillas (2016):

Atención sostenida: este tipo de atención se define como el mantenimiento del foco atencional durante un tiempo prolongado ante una tarea presentada permitiendo el óptimo procesamiento de la información. Una de las características primordial de la atención sostenida es el estado de alerta o arousal y la vigilancia que incrementan la concentración, sin embargo, si existe la presencia de un estímulo distractor se disminuirá el nivel de atención o intensidad hasta finalizar la tarea.

- Atención selectiva: es la capacidad de centrar la atención en un estímulo determinado inhibiendo los estímulos distractores, este proceso se lleva a cabo gracias a la selección correcta del estímulo relevante. La atención selectiva se lleva a cabo en dos mementos: el primero en elegir apropiadamente el estímulo y el segundo en preparar la respuesta basándose en la información proporcionada por el estímulo elegido inicialmente.

- Atención dividida: este tipo de atención hace referencia a la capacidad de atender varios estímulos a la vez y estos procesos de llega a cabo con la misma eficacia en las diferentes tareas. Así mismo está encargada de preparar 
respuestas a los múltiples estímulos que solicitan nuestra atención para ejecutar las distintas tareas de forma consecutiva.

- $\quad$ Según el control de voluntad por Luria (1997):

- Atención voluntaria: como su nombre lo indica es la capacidad de concentración de forma voluntaria, aunque el estímulo no sea interesante o intenso. En este tipo de atención implica de control y concentración voluntaria seleccionando exclusivamente un estímulo de varios.

- Atención involuntaria: este es equivalente al reflejo de orientación, es decir, cuando un estímulo llama nuestra atención de forma involuntaria. La atención involuntaria se caracteriza por ser pasiva debido a que no se ejerce ningún tipo intención, refiriéndose a que simplemente atiende a estímulos nuevos de manera inesperada. Esta encargada de percibir estímulos externos que no dependan de la voluntad propia del sujeto.

Factores que influyen en la atención

Según Castillo (2015) los factores que influyen en la atención son factores internos y factores externos.

\section{Factores internos}

- $\quad$ Intensidad: capacidad y fuerza del estímulo para llamar la atención del sujeto.

- $\quad$ Novedad: un estímulo nuevo y diferente fuera de la cotidianidad.

- Tamaño: hace referencia a la grandeza del estímulo el cual llamara más la atención que un estímulo pequeño.

- Repetición: la presencia repetitiva del estímulo disminuirá el nivel de atención provocando una pérdida de interés.

- $\quad$ Cambio: el movimiento inesperado del estímulo atraerá la atención del sujeto.

- $\quad$ Factores internos

- Emociones: hace referencia principalmente a las emociones del sujeto el mismo que llamara la atención.

- Intereses: en este factor involucra los gustos e interés de cada persona hacia el estímulo determinado.

- Estado orgánico: hace referencia a las necesidades del ser humano y la satisfacción de estas.

\section{Desempeño académico}

El desempeño académico se define como el esfuerzo aplicado en la institución para el cumplimiento de sus metas y objetivos, en el cual el estudiante tenga la capacidad de desarrollar aptitudes ante la sociedad mediante los conocimientos adquiridos anteriormente (Cordero et al., 2016). En el proceso de desempeño académico es necesario 
el desarrollo de destrezas, habilidades, intereses e ideales que permitan obtener aprendizajes significativos en el cual a futuro se representaran como éxitos y metas alcanzadas (Puerta \& Gelves, 2017).

El rendimiento o desempeño académico es considerado como el fruto de la motivación, la influencia, la voluntad y la capacidad de los alumnos además del entorno donde aprenden; también es importante mencionar algunos elementos para mejorar el rendimiento como: el mejoramiento del equipamiento, tener presente la constante conexión entre el aprendizaje teórico y práctico, un correcto asesoramiento, un cargo de trabajo balanceado y un continuo seguimiento de los estudiantes (Blanz, 2014).

También es importante considerar el papel docente principalmente que ofrezcan un aprendizaje de calidad, saber identificar el estilo de aprendizaje de sus alumnos, poseer habilidades comunicativas para crear estrategias educativas de intervención guiadas al mejoramiento de aprendizajes (Suárez et al., 2017). Los docentes tienen la responsabilidad de planificar sus clases de manera que atraiga la atención de sus estudiantes e incentive a la participación para evitar efectos como la desatención, desinterés y aburrimiento que pueden producir consecuencias en el desempeño académico del educando en la institución (Edel, 2003).

El rendimiento académico está relacionado con la percepción académica que hace referencia a las creencias del alumno acerca de las habilidades que posee como las habilidades sociales, la capacidad intelectual, las estrategias de trabajo, la resistencia y la práctica educativa que aportan en el rendimiento académico. Por consiguiente, se puede decir que los estudiantes que consideran esforzarse arduamente en sus estudios sienten un alto nivel de satisfacción, además de tener mejores calificaciones demostrando más interés y productividad en las actividades que demandan sus carreras. Por el contrario, estudiantes con calificaciones inferiores tienden a experimentar sentimientos de tristeza, baja autoestima, vergüenza y depresión (Perry et al., 2005).

Existen dos variables que influyen significativamente en el rendimiento académico los mismos que son la motivación y el tiempo dedicado al estudio, es decir, mientras más tiempo se emplee en las actividades de la universidad aportaran de manera positiva a un mejor desempeño estudiantil (Nonis \& Hudson, 2010).

\section{Desempeño académico durante la pandemia covid-19}

Durante la emergencia sanitaria mundial el ministerio de educación ha tomado medidas para continuar el proceso de educación, en la que se ha incorporado el uso de la tecnología para el desarrollo de las actividades académicas. Esta nueva modalidad virtual ofrece una serie de utilidades al momento de adquirir nuevos conocimientos, sin embargo, se debe tener en cuenta que un excelente desempeño académico no requiere solo de obtener 
medios tecnológicos sino también de los actos de responsabilidad del estudiante universitario mucho más durante la modalidad online ya que requiere la participación del docente y en gran parte de su aprendizaje autónomo (Valdivieso, 2020).

En el aprendizaje de modalidad virtual ha presentado manifestaciones en los estudiantes como el estrés, desmotivación, desinterés, desatención, ansiedad y principalmente alteraciones en el desempeño académico. Tanto docentes como estudiantes han tenido que someterse al proceso de adaptación al nuevo contexto de educación mediante la implementación de estrategias virtuales para llevar a cabo el proceso de enseñanza aprendizaje (León, 2020).

Valdivieso (2020) anuncia sobre las dificultades culturales que se suman a la pandemia en la cual se refiere a los estudiantes que no prestaban atención de sus clases presenciales asume que menos lo harán durante las clases online.

\section{Factores que influyen en el desempeño académico}

Para Castillo (2015) existen tres factores que pueden influir en el desempeño académico:

- Factores socioculturales: hace referencia a la carencia de recursos económicos y el nivel de educación de los padres que traen consecuencias en la formación académica de sus hijos al no contar con el conocimiento necesario para ayudar en el cumplimiento y reforzamiento de la enseñanza impartida por el docente.

- $\quad$ Factores pedagógicos: estos factores se enfocan principalmente a los métodos y estrategias que los docentes utilizan para impartir sus clases que abren una brecha al desinterés y susceptibilidad del estudiante ante las distracciones.

- Factores familiares: hace referencia a las prácticas de crianza, las relaciones padres e hijos, la afectividad y el interés de los padres en la educación de sus hijos.

\section{Evaluación del desempeño académico}

La evaluación del desempeño académico según el Ministerio de Educación pretende medir las actitudes y aptitudes el educando que ha obtenido como resultado del proceso de enseñanza-aprendizaje. Esta evaluación de conocimientos se hace mediante el uso de instrumentos que evidencien las destrezas y habilidades adquiridas en el aula de clase. Para evidenciar el desempeño académico se expresa mediante la calificación cualitativa y cuantitativa de las capacidades del alumno.

En estudiantes universitarios la atención no es suficientemente valorada ya que al ser adultos se considera como un acto de responsabilidad autónoma su desempeño académico en cual pueden interferir factores que conllevan a que los estudiantes no logren focalizar 
su atención al estímulo correcto. Principalmente en esta nueva modalidad virtual en las que se presentan constantemente desigualdades educativas que provocan dificultades para mantener su atención en sus clases debido a la presencia de una serie de estímulos tanto internos como externos que interfieren en el aprendizaje y desempeño de los estudiantes universitarios.

\section{Metodología}

\section{Participantes}

Se obtuvo una muestra de 110 estudiantes de la Universidad Técnica de Ambato (ver tabla 1). La selección de muestra hizo mediante un muestreo intencional de tipo no probabilístico. Para su selección se tuvo en cuenta algunas características como: accesibilidad a internet, que los estudiantes asistan de manera regular a clases online y que tengan un computador a su disposición.

\section{Tabla 1}

Participantes de la investigación

\begin{tabular}{lcc}
\hline \multicolumn{1}{c}{ Genero } & Participantes & Porcentaje \\
\hline Masculino & 28 & $25 \%$ \\
Femenino & 82 & $75 \%$ \\
\hline Total & 110 & $100 \%$ \\
\hline
\end{tabular}

Elaborado por: Verónica Andreina Caizaguano Azogue

\section{Instrumentos}

Para la presente investigación se utilizó la prueba de atención d2 de Rolf Brickenkamp para valorar la atención sostenida. Para su aplicación se utilizó una herramienta en línea llamada Google Forms que permitió virtualizar la prueba mediante el uso de imágenes y botones de selección. Se presentó 15 secciones, en la primera sección se rellenan los datos informativos del estudiante (nombre y apellido, edad, género, curso, paralelo y fecha de evaluación). Desde la segunda sección hasta la quinceava sección corresponde a la prueba, cada sección está dividida en 5 áreas (1-10,11-20, 21-30, 31-40, 41-47) representando cada línea de trabajo la misma que está constituida de letras d y p (estímulos relevantes y estímulos irrelevantes).

La segunda variable se evaluó mediante un cuestionario para el desempeño académico realizado mediante una operacionalizacion de variables que fue validad por un profesional experto. El cuestionario consta de 15 dimensiones con 15 preguntas que 
ayudan a obtener información sobre la percepción que tiene el estudiante de su desempeño académico.

\section{Métodos}

Este estudio es de tipo mixto cualitativo-cuantitativo. Es cualitativo porque se hizo una descripción e interpretación de los datos obtenidos a través de los instrumentos. Y cuantitativo porque se hizo el análisis de los datos estadísticos. La investigación es de nivel descriptivo ya que se hizo una descripción de las características de ambas variables para su operacionalización. Y documental bibliográfico debido a la extensa revisión bibliográfica de artículos, libros y revistas científicas que sustenten el presente estudio.

\section{Resultados}

Resultados de la prueba de atención d2

En el siguiente grafico se presentan los resultados de cada factor de la prueba de atención d2.

\section{Figura 1}

Factores de la prueba de atención d2

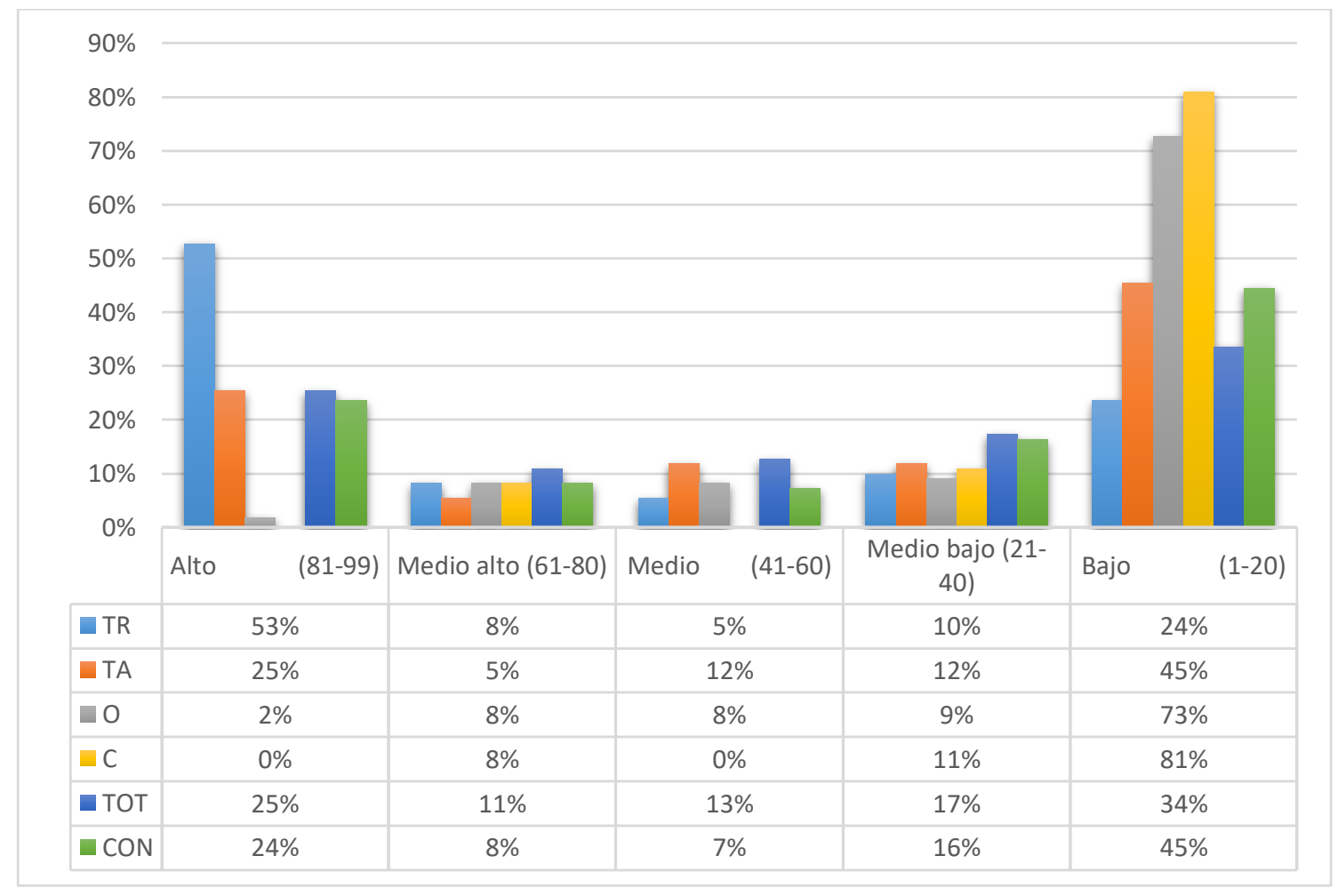

Nota: Resultados de los factores de la prueba de atención d2. Fuente: Estudiantes de la Universidad Técnica de Ambato. Elaborado por: Verónica Andreina Caizaguano Azogue. 
En los resultados obtenidos en la prueba de atención d2 se puede apreciar una predominancia en el nivel bajo con elevados porcentajes en cada uno de los factores correspondientes a la prueba. En el cual se puede decir que los estudiantes presentan dificultades como baja precisión en identificar estímulos relevantes, poca atención y omisión de respuestas correctas, baja capacidad de discriminación, bajo rendimiento de la atención sostenida, fatiga de la atención e ineficiencia en realizar una tarea.

\section{Resultados del Cuestionario para el desempeño académico}

En el siguiente grafico se presentan los resultados de las dimensiones del cuestionario para el desempeño académico.

\section{Figura 2}

\section{Dimensiones del Cuestionario para el desempeño académico}

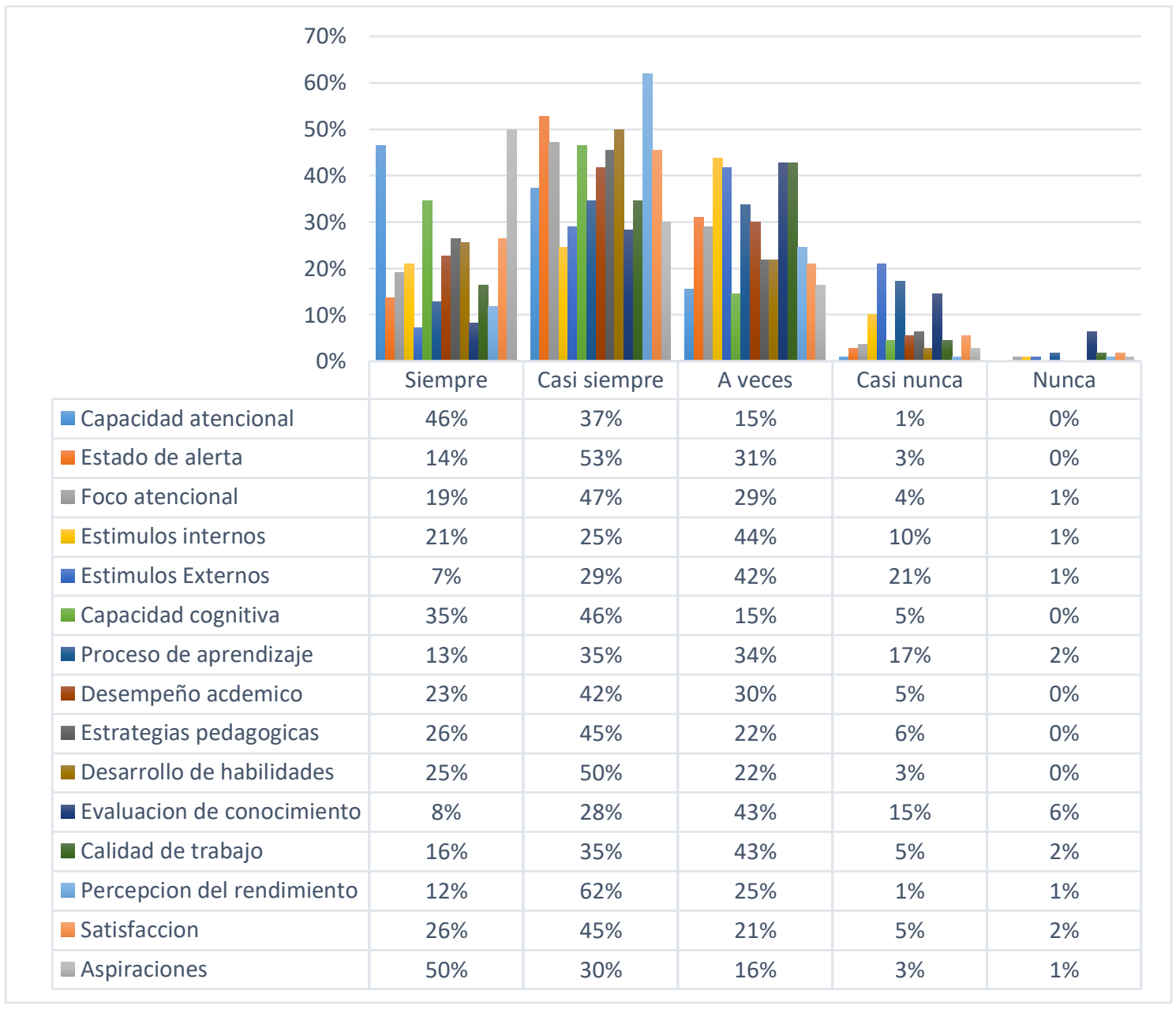

Nota: Resultados de los factores de la prueba de atención d2. Fuente: Estudiantes de la Universidad Técnica de Ambato. Elaborado por: Verónica Andreina Caizaguano Azogue. 
En figura 2 se presentan los resultados del cuestionario para el desempeño académico en cual se pudo evidenciar lo siguiente: los estudiantes consideran que al mantener la atención obtendrán un mejor desempeño académico, frecuentemente se mantienen alertas durante las clases, del mismo modo logran estudiar por iniciativa propia, se distraen fácilmente cuando el contenido de sus clases no son de si interés y cuando perciben algún estimulo externo como el celular, además mencionan la importancia de prestar atención en clases para obtener mejores resultados en las evaluaciones. Por otra parte, los docentes frecuentemente motivan a los estudiantes al aprendizaje mediante el manejo de estrategias pedagógicas, por lo que los estudiantes en su mayoría buscan más información adicional a la proporcionada por el docente, consideran que las evaluaciones virtuales en ocasiones pueden medir con exactitud los conocimientos de los estudiantes, además consideran mantener un buen desempeño académico en sus actividades académicas, por el cual se encuentran satisfechos con los esfuerzos dados en sus estudios para ser un excelente profesional a futuro.

\section{Conclusiones}

- Mediante el análisis de los resultados de la prueba de atención d2 se llevó a las siguientes conclusiones: En el total de respuestas un nivel alto en la velocidad de procesamiento, sin embargo, se obtuvo un nivel bajo en la cantidad de aciertos lo que significa que una baja precisión en la identificación de los estímulos relevantes. En los errores por omisión se obtuvo un nivel bajo y de acuerdo con el manual se puede decir que los evaluados presentan poca atención y omiten respuestas correctas. Con respecto a los errores por comisión presentan un nivel bajo por el cual se deduce una menor capacidad de discriminación de estímulos relevantes. En el índice de efectividad de la prueba presentan un nivel bajo traduciéndose como fatiga atencional y un mal rendimiento en la atención sostenida. Finalmente, en el factor de concentración se evidencia un nivel bajo por lo mismo presentan concentración con ineficiencia en la realización de una tarea.

- Por otra parte, en el desempeño académico se pudo evidenciar que los participantes a pesar de encontrarse en un estado de alerta durante la jornada de clases presentan dificultades en el mantenimiento de la atención por largos periodos de tiempo debido a la presencia de estímulos tanto internos como externos en el ámbito de estudio en el que se encuentran los mismos que repercuten en el desenvolvimiento de los estudiantes en sus actividades académicas. 


\section{Referencias bibliográficas}

Blanz, M. (2014). ¿Cómo se interrelacionan la satisfacción del estudio y el rendimiento académico? Una investigación con estudiantes de programas de Trabajo Social. Revista Europea de Social Work, 281-292.

Boujon, C., \& Quaireau, C. (2004). Atención, aprendizaje y rendimiento académico. Madrid: NARCEA, S.A. DE EDICIONES.

Campabadal, A., Jodar, M., Lubrini, G., Periañez, J., Ríos, M., Muñoz, E., \& Viejo, R. (2008). Neuropsicología de la atención. Neuropsicología de la atención, la memoria y las funciones ejecutivas.

Castillo, C. (2015). La atención en el rendimiento escolar de los niños de tercer grado paralelo " $B$ " de la escuela de educación básica "Benigno Bayencela" del sector San Cayetano bajo de la ciudad de Loja. Loja.

Corbetta, M., \& Shulman, G. (2002). Control de la atención dirigida por objetivos y dirigida por estímulos en el cerebro. Nature Reviews Neuroscience, 201-215.

Cordero, A., Rivera, A., \& Oppenheimer, L. (2016). Desempeño académico universitario: Facultad de Filosofía y Letras de la Universidad Autónoma de Chihuahua. RECIE. Revista Electrónica Científica de Investigación Educativa, 363-671.

Edel, R. (2003). El rendimiento académico: concepto, investigación y desarrollo. REICE. Revista Iberoamericana sobre Calidad, Eficacia y Cambio en Educación.

Esparza, H., \& Casillas, E. (2016). Asociación entre calidad atencional y atención sostenida con las habilidades de análisis visual. Aguascalientes.

Flores, E. (2016). Proceso de la atención y su implicación en el proceso de aprendizaje. Revista Didasc@lia: D\&E. Publicación cooperada entre CEDUT- Las Tunas y CEdEG-Granma, CUBA.

León, M. (2020). Incidencia de la practica de valores en el rendimiento académico de la enseñanza online generado por la pandemia "covid-19" en el tiempo de confinamiento reflexionando desde la Carrera de Educación Básica de la FCJSEUTB, Cantón Babahoyo, Los Ríos. Babahoyo.

Luria, A. (1979). Atención y Memoria. Barcelona.

Nobre, A. (2001). El homúnculo atento: ahora lo ves, ahora no lo ves. Revisiones de neurociencia y bioconducta, 477- 496. 
Nonis, S., \& Hudson, G. (2010). Rendimiento académico de los estudiantes universitarios: influencia del tiempo dedicado a estudiar y trabajar. Revista de educación para empresas, 151-159.

Perry, R., Hall, N., \& Ruthing, J. (2005). Control percibido (académico) y logro académico en la educación superior. En educación superior: Manual de. Springer Países Bajos, 363-436.

Posner, M., \& Petersen, S. (1990). El sistema de atención del cerebro humano. Revisión anual de neurociencia, 25-45.

Puerta, L., \& Gelves, M. (2017). Atención focalizada, sostenida, inhibición y rendimiento académico. Barranquilla.

Raz, A., \& Buhle, J. (2006). Tipologías de redes atencionales. Nature Reviews Neuroscience, 367-379.

Suárez, E., Suárez, E., \& Pérez, E. (2017). Análisis de los factores asociados al rendimiento académico de estudiantes de un curso de informática. Revista de Pedagogía, 176-192.

Valdivieso, M. (2020). Percepción de estudiantes universitarios colombianos sobre el efecto del confinamiento por el coronavirus y su rendimiento académico. Revista Espacios, 269-281.

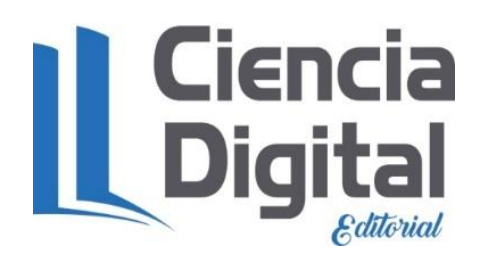


El artículo que se publica es de exclusiva responsabilidad de los autores y no necesariamente reflejan el pensamiento de la Revista Conciencia Digital.

\section{Ciencia}

El artículo queda en propiedad de la revista y, por tanto, su publicación parcial y/o total en otro medio tiene que ser autorizado por el director de la Revista Conciencia Digital.
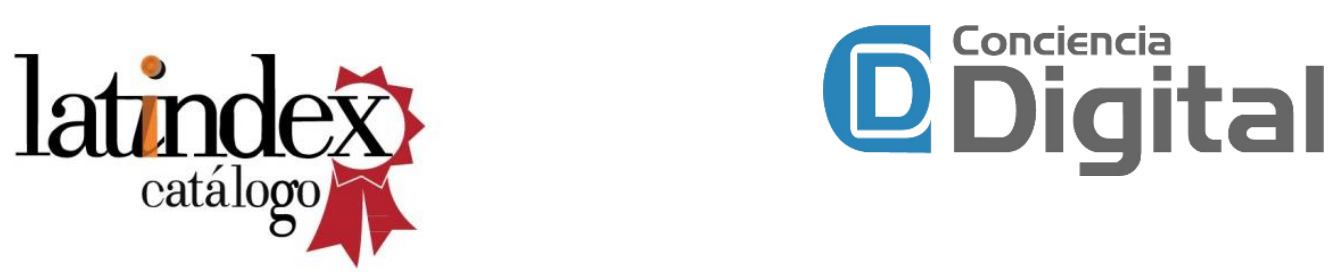

Indexaciones

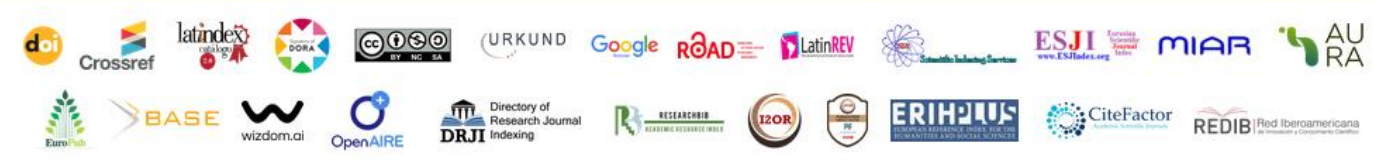

